\title{
TEVATRON BEAM LIFETIMES AT INJECTION USING THE SHOT DATA ANALYSIS SYSTEM*
}

\author{
A. Xiao", T. Bolshakov, P. Lebrun, E. McCrory, V. Papadimitriou, A. J. Slaughter \\ FNAL, Batavia, IL 60510, U.S.A.
}

\begin{abstract}
The purpose of the Shot Data Acquisition and Analysis (SDA) system is to provide summary data on the Fermilab RunII accelerator complex and provide related software for detailed analyses. In this paper, we discuss such a specific analysis on Tevatron beam lifetimes at injection. These results are based on SDA data, tools and methodology. Beam lifetime is one of our most important diagnostics. An analysis of it can give information on intra beam scattering, aperture limitations, instabilities and most importantly beam-beam effects. Such an analysis gives us a better understanding of our machine, and will lead to an improved performance in the future.
\end{abstract}

\section{INTRODUCTION}

For a large scientific facility like the Fermilab RunII accelerator complex, there is a huge amount of data from various control systems and measurement devices. The SDA system is designed to organize these data and provide tools and methodology for later review and analyses [1,2].

In this paper we present a specific application of SDA on beam lifetime at injection. One of the limiting factors in collider performance is the proton lifetime at $150 \mathrm{GeV}$. We focus on the protons instead of the antiprotons because the proton losses are higher and because of the relatively large proton emittances compared to the ones from antiprotons. Here, we measure the bunch-by-bunch proton lifetimes at different stages of the injection process and document definite hints for adverse beam-beam effects of the antiprotons on the proton beam.

\section{LIFETIME AT INJECTION}

The proton/antiproton injection scheme and relevant events are shown in Figure 1. We have observed that the proton lifetime changes at different injection stages. For example, the lifetime is better after opening the helix and becomes worse as the antiprotons are injected into the machine. This hints at parasitic beam-beam effects and probably physical aperture limitations. To investigate these phenomena, we calculate the proton bunch lifetime at different stages.

\section{General Description of Tevatron Injection}

The Tevatron operates with 36 proton bunches and 36 antiproton bunches circulating in opposite directions. We fill the machine in these steps (Figure 1): Load the 36 proton bunches on the center orbit one by one into 3 trains

*Work supported by DOE under contract DE-AC02-76CH03000 with the U.S. Dept. of Energy.

"xiaoam@fnal.gov separated by $\sim 4.3 \mu$ sec abort gaps; Set the proton bunches onto a helical orbit, so that they will not collide with the injected antiprotons; Load 3 mini trains, each with 4 consecutive antiproton bunches into the abort gaps; Move (cog) the antiproton bunches longitudinally with respect to the proton bunches; Repeat the antiproton injection and cogging two more times. The injection scheme has been illustrated in Figure 2.

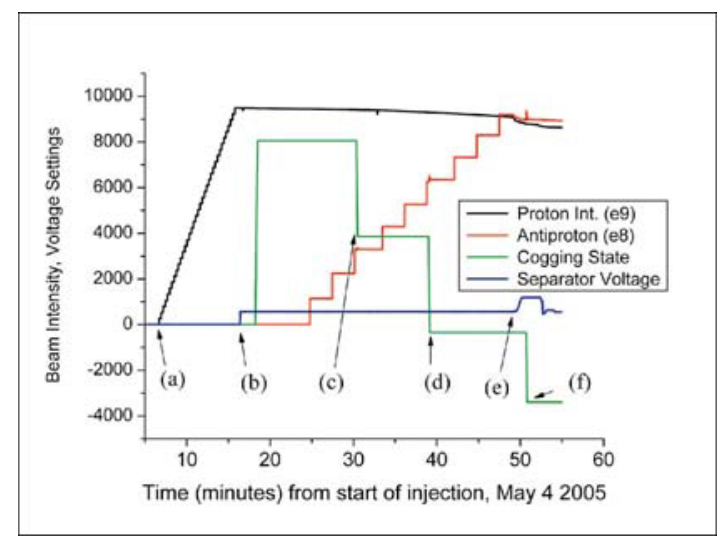

Figure 1: Tevatron Injection: the black line is the proton intensity, the red line, the antiproton intensity, the blue line, separator's voltage, and the green line, the antiproton RF bucket offsets, respectively. The relevant events are (a) start inject proton. (b) open helix (c)/(d)/(f) change of the RF for 1st/2nd/3rd cogging and (e) the start of the ramp.

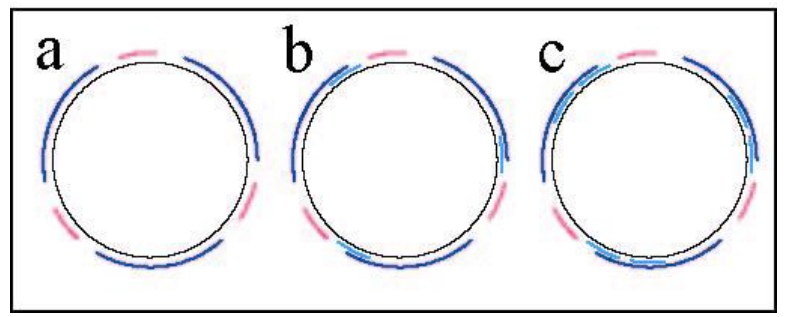

Figure 2: Injection and Cogging. The blue traces are for protons, the magenta and cyan traces are for antiprotons. (a) inject $1^{\text {st }} 3$ mini antiproton bunch trains. (b) cog and inject $2^{\text {nd }} 3$ mini antiproton bunch trains. (c) $\operatorname{cog}$ and inject $3^{\text {rd }} 3$ mini antiproton bunch trains.

\section{Lifetime Calculation}

We calculate the proton bunch lifetimes at each of the above stages, except for the final cogging at full energy.

The lifetime has been calculated for the 5-minute period just before the machine changes to the next stage, for example open helix. It is based on an exponential fit of the bunch intensities for the 5-minute period. Figures 3 
and 4 show the proton lifetimes versus bunch number at different stages. We make the following observations:

- Before opening the helix, the earlier and later injected bunches have shorter lifetime than those in the middle. (Figure 3, top.)

- Before the $1^{\text {st }}$ cogging, there is no lifetime variation for bunches in train 1, but the last 3 bunches in trains 2 and 3 have shorter lifetimes. (Figure 3, bottom.)

- Before the $2^{\text {nd }}$ cogging, the number of proton bunches with shorter lifetime increases and moves forward by 4 bunches. There is now a lifetime variation in train 1 as well. The last 3 bunches in train 1 still have the original lifetime. (Figure 4, top.)

- Before the ramp, the pattern is similar to the previous one, except that it moves forward by another 4 bunches. (Figure 4, bottom.)
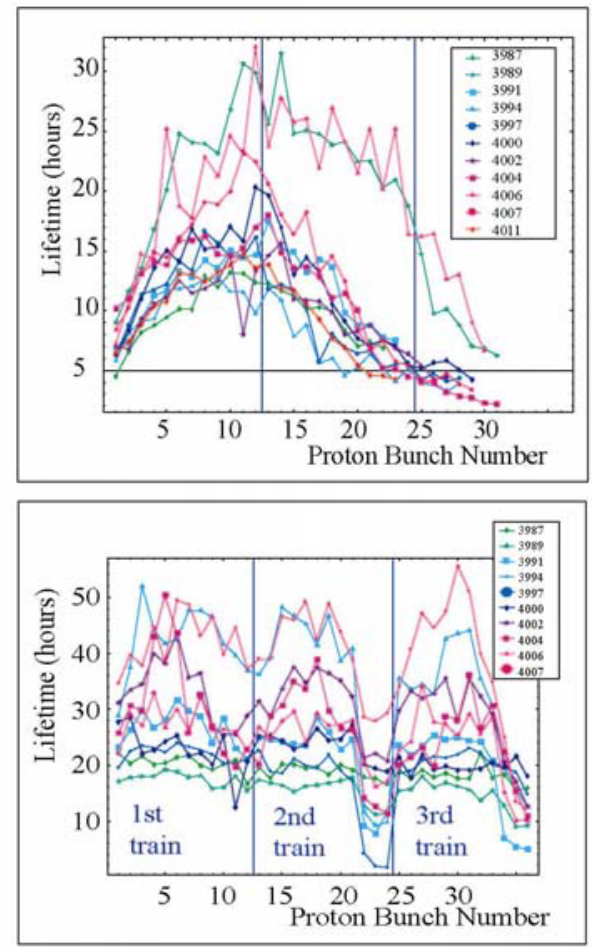

Figure 3: Proton lifetimes for 10 different stores. Top: central orbit. Bottom: before 1st cogging.

\section{Lifetime at Central and Helical Orbit}

The lifetime on the central orbit is shorter for the early and late bunches. The shorter lifetimes for the early bunches could be explained by emittance dilution, which is due to inter beam scattering, and subsequent scraping. To understand the shorter lifetimes for bunches injected last, we calculate the lifetime for each bunch just after its injection as shown in Figure 5. In this figure we see that lifetimes decrease as more protons are injected into the ring, so it could be explained by beam loading effects.

Comparing lifetimes before open helix and before $1^{\text {st }}$ cogging (Figure 3), we see better beam lifetime on the helical orbit. This phenomenon could be explained by two effects: 1) the machine is optimized on the helical orbit and 2) the proton beam orbit is moved away from some physical aperture limitation such as the Lambertson.
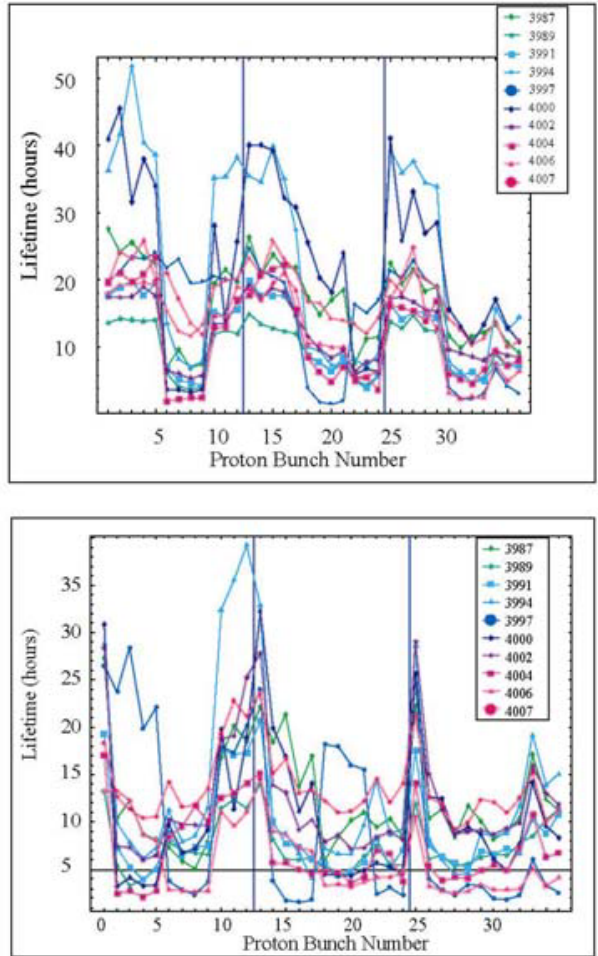

Figure 4: Proton lifetimes for 10 different stores. Top: Before the $2^{\text {nd }}$ cogging. Bottom: before the ramp.

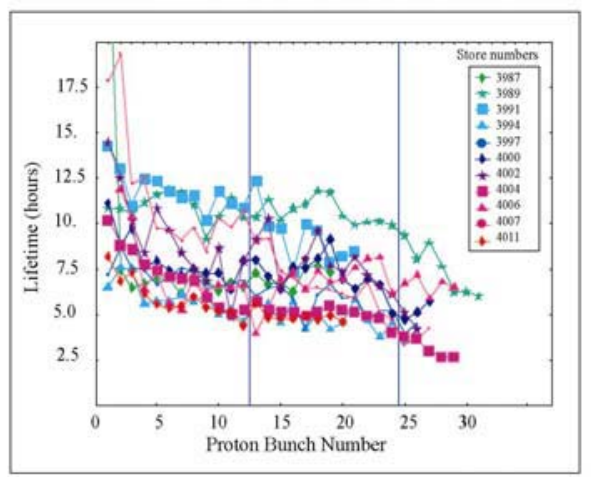

Figure 5: Proton lifetimes at Beginning of Injection

\section{Lifetime at Different Cogging Stages}

The decrease in the proton bunch lifetime as antiproton bunches are injected into the ring is a clear sign of parasitic beam-beam effects. To understand this effect and reveal its location we need a better understanding of the bunch-by-bunch crossing scheme.

The Tevatron has a 6-fold symmetry in its layout. We label the middle of each long straight section as A0 to F0. Table 1 gives the mapping of where each proton bunch train crosses with each antiproton bunch train. Before the $1^{\text {st }}$ cogging the first A1, A2 and A3 antiproton minitrains have been injected into the ring. Since A3 is the last injected one, it has less impact on calculated proton lifetime. As shown in table 1, P1 crosses with A1 at 
regions $\mathrm{C} 0$ and $\mathrm{F} 0$, with $\mathrm{A} 2$ at regions $\mathrm{A} 0$ and $\mathrm{D} 0$. The lifetime of the P1 bunches are all the same, indicating that there are no strong beam-beam effect in these regions (yellow boxes in Table 1). The shorter lifetime of P2 and P3 indicates the possible strong beam-beam effects region is near B0 and E0 (blue boxes in Table 1).

Table 2 shows the detailed proton and antiproton bunch-crossing pattern before the $1^{\text {st }}$ cogging. The fact that bunches 10 to 12 have shorter lifetimes than bunches 1 to 9 indicates that only the highlighted (blue) parasitic crossing point could be the source for this lifetime variation pattern.

The bunch-crossing pattern before the $2^{\text {nd }}$ cogging is shown in Table 3. Now we have the 12 previous injected antiproton bunches in the A1-A3 trains, and the newly injected 8 bunches in $\mathrm{A} 1$ and $\mathrm{A} 2$. If the previous conclusion is correct then bunches 6-9 in P1 and 6-12 in P2 and P3 should now have shorter lifetimes, which is consistent with Figure 4.

Table 1: Bunch Train Crossing Scheme. P and A indicates proton and antiproton, 1-3 indicates the train number

\begin{tabular}{|c|c|c|c|}
\hline A0 & $\mathrm{P} 1 \times \mathrm{A} 2$ & $\mathrm{P} 2 \times \mathrm{A} 3$ & P3 x A1 \\
\hline B0 & $\mathrm{P} 1 \times \mathrm{A} 3$ & $\mathrm{P} 2 \times \mathrm{A} 1$ & P3 x A2 \\
\hline CO & $\mathrm{P} 1 \times \mathrm{A} 1$ & $\mathrm{P} 2 \times \mathrm{A} 2$ & P3 x A3 \\
\hline Do & $\mathrm{P} 1 \times \mathrm{A} 2$ & $\mathrm{P} 2 \times \mathrm{A} 3$ & P3 x A1 \\
\hline E0 & $\mathrm{P} 1 \times \mathrm{A} 3$ & $\mathrm{P} 2 \times \mathrm{A} 1$ & P3 x A2 \\
\hline Fo & $\mathrm{P} 1 \times \mathrm{A} 1$ & P2 x A2 & P3 x A3 \\
\hline
\end{tabular}

Table 2: Proton Bunches Cross with Antiproton Bunches before $1^{\text {st }}$ cogging

\begin{tabular}{|l|l|l|l|l|l|l|}
\hline $12 \times 1$ & $12 \times 2$ & $12 \times 3$ & $12 \times 4$ & & & \\
\hline & $11 \times 1$ & $11 \times 2$ & $11 \times 3$ & $11 \times 4$ & & \\
\hline & & $10 \times 1$ & $10 \times 2$ & $10 \times 3$ & $10 \times 4$ & \\
\hline & & & $9 \times 1$ & $9 \times 2$ & $9 \times 3$ & $9 \times 4$ \\
\hline
\end{tabular}

Table 3: Proton Bunches Cross with Antiproton Bunches before $2^{\text {nd }}$ Cogging

\begin{tabular}{|c|c|c|c|c|c|c|}
\hline \multicolumn{7}{|c|}{ Crossing point before $1^{\text {st }}$ cogging } \\
\hline & & $12 \times 3$ & & & & \\
\hline \multicolumn{7}{|c|}{ Crossing point after $1^{\text {st }}$ cogging } \\
\hline $12 \times 5$ & $12 \times 6$ & $12 \times 7$ & $12 \times 8$ & & & \\
\hline $11 \times 4$ & $11 \times 5$ & $11 \times 6$ & $11 \times 7$ & $11 \times 8$ & & \\
\hline \multicolumn{7}{|l|}{$\ldots$} \\
\hline & & $6 \times 1$ & $6 \times 2$ & $6 \times 3$ & $6 \times 4$ & \\
\hline & & & $5 \times 1$ & $5 \times 2$ & $5 \times 3$ & $5 \times 4$ \\
\hline
\end{tabular}

This analysis of the proton antiproton crossing scheme points to 2 possible parasitic crossing points in $\mathrm{B} 0$ and $\mathrm{E} 0$ region as explaining all the lifetime patterns we observed in Figures 3 and 4 . The s coordinate of the crossing point can be calculated from RF frequency, cogging offset and bunch train structure. It indicates a point 112.9 meters downstream of B0 or E0.

The Tevatron model shown in Figure 6 points to the location $112.9 \mathrm{~m}$ downstream of B0. This is an independent confirmation of the same conclusion from the resonance analysis by Y. Alexahin [3].

It should be noted that this location is not the minimum separation around the Tevatron ring, which indicates that the beam-beam effects also depend on local optical functions and other effects. We have not included antiproton bunch lifetimes in this report since they do stay at injection for a very short time, and it appears that the beam-beam effects on antiproton bunches are not very serious due to smaller bunch emittances. At present the shorter proton bunch lifetimes at injection are not the main limitation to Tevatron performance, and changing the separation is a big task. But the understanding of the problem will help us in the future as antiproton intensities are increased.

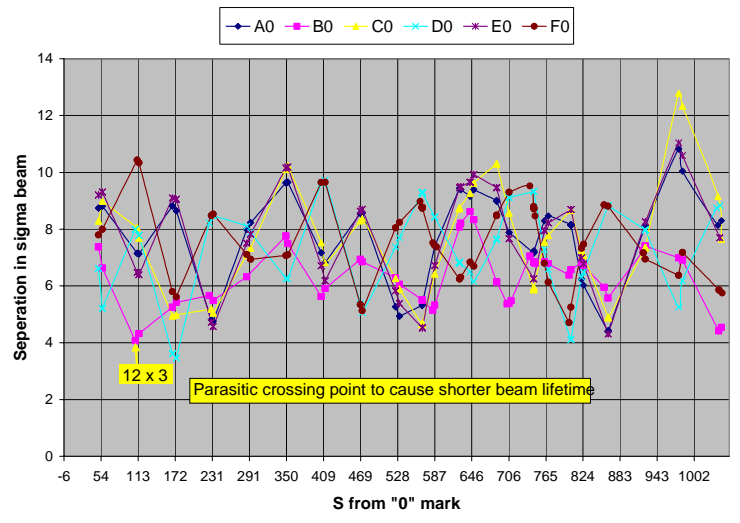

Figure 6: Proton antiproton separation from machine model (MAD).

\section{CONCLUSION}

Using SDA tools, we studied the proton bunch lifetimes at injection. The lifetime varies from stage to stage and from bunch to bunch. From a detailed investigation of the parasitic beam-beam crossing pattern, we found that 2 possible crossing points could have such impact on lifetimes. Comparing with the machine model, we could pinpoint the location to $112.9 \mathrm{~m}$ downstream of B0. This finding agrees with the independent resonance analysis by Y. Alexahin. The location is not the minimum separation point along Tevatron ring, which indicates that the beambeam effects do not only depend on separation, but also on the local optical functions and other effects. The finding improves our understanding of the Tevatron, and may lead to improvements in the future. We also see indications of intra beam scattering effects and beam loading effects.

\section{REFERENCES}

[1] T. B. Bolshakov et al., "SDA-Based Diagnostic and Analysis Tools for Collider Run II,” This conference, FPAT008.

[2] E. McCrory et al., "Fitting the Luminosity Decay in the Tevatron,” This conference, TPAP036.

[3] Y. Alexahin, private communication. 